\title{
Sharp inequalities for hyperbolic functions and circular functions
}

Ling Zhu ${ }^{1 *}$

"Correspondence: zhuling0571@163.com

'Department of Mathematics, Zhejiang Gongshang University, Hangzhou, China

\section{Abstract \\ In this paper, we obtain some new sharp bounds for the exponential functions whose powers involve hyperbolic functions and circular functions, respectively.}

MSC: Primary 26D05; 26D15; secondary 33B10

Keywords: Inequalities; Hyperbolic functions; Circular functions

\section{Introduction}

In [1], Stolarsky obtained the bounds for the exponential function whose power involves a hyperbolic function.

Theorem 1 Let $x>0$. Then

$$
1<e^{x \operatorname{coth} x-1}<\cosh x
$$

On the other hand, Pittenger [2] and Stolarsky [3] got the lower bound for the function $e^{x \operatorname{coth} x-1}$ as follows.

Theorem 2 Let $x>0$. Then

$$
\left(\cosh \frac{2 x}{3}\right)^{\frac{3}{2}}<e^{x \operatorname{coth} x-1}
$$

In fact, Zhu [4] and Kouba [5] showed a new sharp lower bound for the function $e^{x \operatorname{coth} x-1}$ as follows.

Theorem 3 Let $x>0$. Then

$$
\left(\frac{2(\cosh x)^{\frac{6}{5}}+1}{3}\right)^{\frac{5}{6}}<e^{x \operatorname{coth} x-1}
$$

Zhu [6] proved

$$
\left(\cosh \frac{2 x}{3}\right)^{\frac{3}{2}}<\left(\frac{2(\cosh x)^{\frac{6}{5}}+1}{3}\right)^{\frac{5}{6}}, \quad x>0
$$

to illustrate that the inequality (1.3) is stronger than (1.2).

(c) The Author(s) 2019. This article is distributed under the terms of the Creative Commons Attribution 4.0 International License (http://creativecommons.org/licenses/by/4.0/), which permits unrestricted use, distribution, and reproduction in any medium, provided you give appropriate credit to the original author(s) and the source, provide a link to the Creative Commons license, and indicate if changes were made. 
It should be pointed out that the paper of Yang et al. [7] has made a great deal of improvement on inequality (1.2). The subject of the present paper is to further study the inequality (1.3), and to obtain the following results.

Theorem 4 Let $p \neq 0, p_{1}=(\ln (3 / 2)) /(\ln (e / 2))=1.3214 \ldots$, and $x \in(0,+\infty)$. Then we have

(i) when $p \in[2,+\infty)$, the double inequality

$$
e^{p(x \operatorname{coth} x-1)}<\frac{2 \cosh ^{p} x+1}{3}<\frac{2}{3}\left(\frac{e}{2}\right)^{p} e^{p(x \operatorname{coth} x-1)}
$$

holds, where the constants 1 and $(2 / 3)(e / 2)^{p}$ are the best possible;

(ii) when $p \in(-\infty, 6 / 5]$, we have

(a) if $p \in(0,6 / 5]$, the double inequality (1.5) reverses, that is,

$$
\frac{2}{3}\left(\frac{e}{2}\right)^{p} e^{p(x \operatorname{coth} x-1)}<\frac{2 \cosh ^{p} x+1}{3}<e^{p(x \operatorname{coth} x-1)}
$$

holds, where the constants $(2 / 3)(e / 2)^{p}$ and 1 are the best possible;

(b) if $p \in(-\infty, 0)$, the left-hand side of the double inequality (1.5) holds too;

(iii) when $p \in(6 / 5,2)$, we have

(c) if $p \in\left[p_{1}, 2\right)$, the left-hand side of inequality (1.5) holds;

(d) if $p \in\left(6 / 5, p_{1}\right)$, the left-hand side of inequality (1.6) holds.

As straightforward consequences of Theorem 4, Theorem 5 which is due to Kouba [5] may be derived immediately.

Theorem 5 Let $p \neq 0$, and $p_{1}=(\ln (3 / 2)) /(\ln (e / 2))=1.3214 \ldots$ Then

(1) the inequality

$$
\frac{2 \cosh ^{p} x+1}{3}<e^{p(x \operatorname{coth} x-1)}
$$

holds for all $x \in(0,+\infty)$ if and only if $p \in(0,6 / 5]$;

(2) the inequality (1.7) reverses for all $x \in(0,+\infty)$ if and only if $p \in(-\infty, 0) \cup\left[p_{1},+\infty\right)$.

The analog of Theorem 4 for the circular functions is the following result.

Theorem 6 Let $p \neq 0, p_{2}=\ln 3=1.0986 \ldots$, and $x \in(0, \pi / 2)$. Then we have

(i) when $p \in[6 / 5,+\infty)$, the double inequality

$$
e^{p(x \cot x-1)}<\frac{2 \cos ^{p} x+1}{3}<\left(\frac{e^{p}}{3}\right) e^{p(x \cot x-1)}
$$

holds, where the constants 1 and $e^{p} / 3$ are the best possible;

(ii) when $p \in(-\infty, 1]$, we have

(a) if $p \in(0,1]$, the double inequality (1.8) reverses, that is,

$$
\left(\frac{e^{p}}{3}\right) e^{p(x \cot x-1)}<\frac{2 \cos ^{p} x+1}{3}<e^{p(x \cot x-1)}
$$

holds, where the constants $e^{p} / 3$ and 1 are the best possible; 
(b) if $p \in(-\infty, 0)$, the double inequality (1.8) holds too;

(iii) when $p \in(1,6 / 5)$, we have

(c) if $p \in\left(p_{2}, 6 / 5\right)$, the right-hand side of inequality (1.8) holds;

(d) if $p \in\left(1, p_{2}\right]$, the right-hand side of inequality (1.9) holds.

The following result which is due to Yang et al. [7] is a straightforward consequence of Theorem 6.

Theorem 7 Let $p \neq 0$, and $p_{2}=\ln 3=1.0986 \ldots$ Then

(A) the inequality

$$
e^{p(x \cot x-1)}<\frac{2 \cos ^{p} x+1}{3}
$$

holds for all $x \in(0, \pi / 2)$ if and only if $p \in(-\infty, 0) \cup[6 / 5,+\infty)$;

(B) the inequality (1.10) reverses for all $x \in(0, \pi / 2)$ if and only if $p \in\left(0, p_{2}\right]$.

\section{Lemmas}

Lemma 1 ([8]) For $-\infty<a<b<\infty$, let $f, g:[a, b] \rightarrow \mathbb{R}$ be continuous functions that are differentiable on $(a, b)$, with $f(a)=g(a)=0$ or $f(b)=g(b)=0$. Assume that $g^{\prime}(x) \neq 0$ for each $x$ in $(a, b)$. If $f^{\prime} / g^{\prime}$ is increasing (decreasing) on $(a, b)$, then so is $f / g$.

Lemma 2 ([9]) Let $A(x)=\sum_{n=0}^{\infty} a_{n} x^{n}$ and $B(x)=\sum_{n=0}^{\infty} b_{n} x^{n}$ be two real power series converging on $(0, r)(r>0)$ with $b_{n}>0$ for all $n$. If the sequence $\left\{a_{n} / b_{n}\right\}$ is increasing (decreasing) for all $n$, then the function $A(x) / B(x)$ is also increasing (decreasing) on $(0, r)$.

Lemma 3 Let $l(x)$ be defined by

$$
l(x)=\frac{\ln \frac{\sinh 2 x-2 x}{4(x \cosh x-\sinh x)}}{\ln \cosh x}
$$

Then $l(x)$ is strictly increasing from $(0,+\infty)$ onto $(1 / 5,1)$.

Proof Let

$$
l(x)=\frac{\ln \frac{\sinh 2 x-2 x}{4(x \cosh x-\sinh x)}}{\ln \cosh x}=: \frac{f(x)}{g(x)}=\frac{f(x)-f\left(0^{+}\right)}{g(x)-g\left(0^{+}\right)},
$$

where

$$
f(x)=\ln \frac{\sinh 2 x-2 x}{4(x \cosh x-\sinh x)}, \quad g(x)=\ln \cosh x
$$

Then

$$
\begin{aligned}
f^{\prime}(x) & =\frac{2 \cosh 2 x-2}{\sinh 2 x-2 x}+\frac{x \sinh x}{\sinh x-x \cosh x} \\
& =\sinh x\left(\frac{4 \sinh x}{\sinh 2 x-2 x}+\frac{x}{\sinh x-x \cosh x}\right), \\
g^{\prime}(x) & =\frac{\sinh x}{\cosh x}
\end{aligned}
$$


We compute

$$
\begin{aligned}
\frac{f^{\prime}(x)}{g^{\prime}(x)} & =\frac{\sinh x\left(\frac{4 \sinh x}{\sinh 2 x-2 x}+\frac{x}{\sinh x-x \cosh x}\right)}{\frac{\sinh x}{\cosh }} \\
& =\left(\frac{x}{\sinh x-x \cosh x}+4 \frac{\sinh x}{-2 x+\sinh 2 x}\right) \cosh x \\
& =(\cosh x) \frac{-4 \sinh ^{2} x-x \sinh 2 x+2 x^{2}+4 x \cosh x \sinh x}{(-2 x+\sinh 2 x)(-\sinh x+x \cosh x)} \\
& =\frac{\left(2 x^{2} \cosh x+2 x \cosh { }^{2} x \sinh x-4 \cosh x \sinh ^{2} x\right)}{\left.-\left(2 x^{2} \cosh x-2 x \cosh \right)^{2} x \sinh x-2 x \sinh x+2 \cosh ^{2} \sinh ^{2} x\right)} \\
& =\frac{\left(\cosh x-\cosh 3 x+\frac{1}{2} x \sinh x+\frac{1}{2} x \sinh 3 x+2 x^{2} \cosh x\right)}{-\left(\frac{1}{2} \cosh 3 x-\frac{1}{2} \cosh x-\frac{5}{2} x \sinh x-\frac{1}{2} x \sinh 3 x+2 x^{2} \cosh x\right)} \\
& =\frac{2 \cosh x-2 \cosh 3 x+x \sinh x+x \sinh 3 x+4 x^{2} \cosh x}{-\cosh 3 x+\cosh x+5 x \sinh x+x \sinh 3 x-4 x^{2} \cosh x} \\
& :=\frac{A(x)}{B(x)},
\end{aligned}
$$

where

$$
\begin{aligned}
& A(x)=2 \cosh x-2 \cosh 3 x+x \sinh x+x \sinh 3 x+4 x^{2} \cosh x, \\
& B(x)=-\cosh 3 x+\cosh x+5 x \sinh x+x \sinh 3 x-4 x^{2} \cosh x .
\end{aligned}
$$

Then

$$
\begin{aligned}
& A(x)=\sum_{n=2}^{+\infty} \frac{6(n-2) 3^{2 n}+16 n^{2}+26 n+12}{(2 n+2) !} x^{2 n+2}=: \sum_{n=2}^{+\infty} a_{n} x^{2 n+2}, \\
& B(x)=\sum_{n=2}^{+\infty} \frac{3(2 n-1) 3^{2 n}-16 n^{2}-14 n+3}{(2 n+2) !} x^{2 n+2}=: \sum_{n=2}^{+\infty} b_{n} x^{2 n+2},
\end{aligned}
$$

where

$$
\begin{aligned}
& a_{n}=\frac{6(n-2) 3^{2 n}+16 n^{2}+26 n+12}{(2 n+2) !}, \\
& b_{n}=\frac{3(2 n-1) 3^{2 n}-16 n^{2}-14 n+3}{(2 n+2) !} .
\end{aligned}
$$

We can obtain

$$
\frac{a_{n}}{b_{n}}=2 \frac{3(n-2) 3^{2 n}+8 n^{2}+13 n+6}{3(2 n-1) 3^{2 n}-16 n^{2}-14 n+3}=: 2 s_{n}, \quad n \geq 2
$$

Now we will prove that $\left\{s_{n}\right\}_{n \geq 2}$ is strictly increasing, which means

$$
s_{n}<s_{n+1} \quad \Longleftrightarrow \quad h(n) 3^{2 n}+32 n^{2}+112 n+81>0,
$$


where

$$
h(n)=: 81 \cdot 3^{2 n}-\left(256 n^{3}+224 n^{2}+112 n+162\right)>0
$$

for $n \geq 2$ due to

$$
\begin{aligned}
& h(n+1)-9 h(n)=2048 n^{3}+1024 n^{2}-320 n+704 \\
& \quad=20,544+28,352(n-2)+13,312(n-2)^{2}+2048(n-2)^{3}>0
\end{aligned}
$$

for $n \geq 2$ and $h(2)=3231>0$. This leads to $s_{n}<s_{n+1}$ for $n \geq 2$. So $\left\{a_{n} / b_{n}\right\}_{n \geq 2}$ is strictly increasing. By Lemma 2, we know that $A(x) / B(x)=f^{\prime}(x) / g^{\prime}(x)$ is strictly increasing on $(0,+\infty)$. Then $l(x)=f(x) / g(x)$ is strictly increasing on $(0,+\infty)$ by Lemma 1 .

Since

$$
\lim _{x \rightarrow 0^{+}} \frac{\ln \frac{\sinh 2 x-2 x}{4(x \cosh x-\sinh x)}}{\ln \cosh x}=\frac{1}{5}, \quad \lim _{x \rightarrow+\infty} \frac{\ln \frac{\sinh 2 x-2 x}{4(x \cosh x-\sinh x)}}{\ln \cosh x}=1
$$

this completes the proof of Lemma 3.

Lemma 4 Let $x>0, B_{2 n}$ be the even-indexed Bernoulli numbers (see [10]). Then the following power series expansions:

$$
\begin{aligned}
& \tan x=\sum_{n=1}^{\infty} \frac{2^{2 n}-1}{(2 n) !} 2^{2 n}\left|B_{2 n}\right| x^{2 n-1}, \\
& \sec ^{2} x=\sum_{n=1}^{\infty} \frac{2^{2 n}\left(2^{2 n}-1\right)(2 n-1)}{(2 n) !}\left|B_{2 n}\right| x^{2 n-2}, \\
& \tan x \sec ^{2} x=\sum_{n=2}^{\infty} \frac{2^{2 n}\left(2^{2 n}-1\right)(2 n-1)(n-1)}{(2 n) !}\left|B_{2 n}\right| x^{2 n-3}
\end{aligned}
$$

hold for all $x \in(-\pi / 2, \pi / 2)$.

Proof The power series expansion (2.2) can be found in [11, equations 1.3.1.4(3)]. By (2.2) we have

$$
(\sec x)^{2}=(\tan x)^{\prime}=\sum_{n=1}^{\infty} \frac{2^{2 n}\left(2^{2 n}-1\right)(2 n-1)}{(2 n) !}\left|B_{2 n}\right| x^{2 n-2}, \quad|x|<\frac{\pi}{2},
$$

and

$$
\begin{aligned}
\tan x \sec ^{2} x & =\frac{1}{2}\left(\sec ^{2} x\right)^{\prime} \\
& =\frac{1}{2} \sum_{n=2}^{\infty} \frac{2^{2 n}\left(2^{2 n}-1\right)(2 n-1)(2 n-2)}{(2 n) !}\left|B_{2 n}\right| x^{2 n-3} \\
& =\sum_{n=2}^{\infty} \frac{2^{2 n}\left(2^{2 n}-1\right)(2 n-1)(n-1)}{(2 n) !}\left|B_{2 n}\right| x^{2 n-3}, \quad|x|<\frac{\pi}{2} .
\end{aligned}
$$


Lemma $5([12,13])$ Let $B_{2 n}$ be the even-indexed Bernoulli numbers. Then

$$
\frac{(2 n+2)(2 n+1)\left(2^{2 n-1}-1\right)}{\pi^{2}\left(2^{2 n+1}-1\right)}<\frac{\left|B_{2 n+2}\right|}{\left|B_{2 n}\right|}<\frac{(2 n+2)(2 n+1)\left(2^{2 n}-1\right)}{\pi^{2}\left(2^{2 n+2}-1\right)}
$$

holds for $n=1,2, \ldots$.

Lemma 6 Let $z(x)$ be defined by

$$
z(x)=\frac{\ln \frac{2 x-\sin 2 x}{4(\sin x-x \cos x)}}{\ln \cos x}
$$

Then $z(x)$ is strictly decreasing from $(0, \pi / 2)$ onto $(0,1 / 5)$.

Proof Let

$$
z(x)=\frac{\ln \frac{2 x-\sin 2 x}{4(\sin x-x \cos x)}}{\ln \cos x}=\frac{p(x)}{q(x)}=\frac{p(x)-p\left(0^{+}\right)}{q(x)-q\left(0^{+}\right)},
$$

where

$$
p(x)=\ln \frac{2 x-\sin 2 x}{4(\sin x-x \cos x)}, \quad q(x)=\ln \cos x .
$$

Since

$$
p^{\prime}(x)=(\sin x)\left[\frac{4 \sin x}{2 x-\sin 2 x}-\frac{x}{\sin x-x \cos x}\right], \quad q^{\prime}(x)=-\frac{\sin x}{\cos x}
$$

we have

$$
\begin{aligned}
\frac{p^{\prime}(x)}{q^{\prime}(x)} & =\frac{x \cos x}{\sin x-x \cos x}-\frac{2 \sin x \cos x}{x-\cos x \sin x} \\
& =\frac{x^{2} \cos x+x \cos ^{2} x \sin x-2 \cos x \sin ^{2} x}{-x^{2} \cos x+x \cos ^{2} x \sin x+x \sin x-\cos x \sin ^{2} x} \\
& =\frac{\left(x^{2} \cos x+x \cos ^{2} x \sin x-2 \cos x \sin ^{2} x\right) / \cos ^{3} x}{\left(-x^{2} \cos x+x \cos ^{2} x \sin x+x \sin x-\cos x \sin ^{2} x\right) / \cos ^{3} x} \\
& =\frac{x^{2} \sec ^{2} x+x \tan x-2 \tan ^{2} x}{-x^{2} \sec ^{2} x+x \tan x+x \tan x \sec ^{2} x-\tan ^{2} x} \\
& =: \frac{C(x)}{D(x)} .
\end{aligned}
$$

From Lemma 4 we obtain

$$
\begin{aligned}
C(x) & =x^{2} \sec ^{2} x+x \tan x-2 \tan ^{2} x \\
& =\sum_{n=3}^{\infty}\left[\frac{(2 n)\left(2^{2 n}-1\right)\left|B_{2 n}\right|}{(2 n) !}-\frac{8\left(2^{2 n+2}-1\right)(2 n+1)\left|B_{2 n+2}\right|}{(2 n+2) !}\right] 2^{2 n} x^{2 n} \\
& =: \sum_{n=3}^{\infty} c_{n} x^{2 n}
\end{aligned}
$$




$$
\begin{aligned}
D(x) & =-x^{2} \sec ^{2} x+x \tan x+x \tan x \sec ^{2} x-\tan ^{2} x \\
& =\sum_{n=3}^{\infty}\left[\frac{2(2 n+1)\left(2^{2 n+2}-1\right)\left|B_{2 n+2}\right|}{(2 n+2) !}-\frac{\left(2^{2 n}-1\right)\left|B_{2 n}\right|}{(2 n) !}\right](n-1) 2^{2 n+1} x^{2 n} \\
& =: \sum_{n=3}^{\infty} d_{n} x^{2 n} .
\end{aligned}
$$

We consider the monotonicity of $C(x) / D(x)$, and compute that

$$
\frac{c_{n}}{d_{n}}=\frac{1}{2(n-1)} \frac{(n+1)(2 n)\left(2^{2 n}-1\right)\left|B_{2 n}\right|-4\left(2^{2 n+2}-1\right)\left|B_{2 n+2}\right|}{\left(2^{2 n+2}-1\right)\left|B_{2 n+2}\right|-(n+1)\left(2^{2 n}-1\right)\left|B_{2 n}\right|} .
$$

Then for $n \geq 3$

$$
\begin{aligned}
\frac{c_{n}}{d_{n}} & >\frac{c_{n+1}}{d_{n+1}} \Longleftrightarrow \\
\frac{L}{M} & =: \frac{(n+1)(2 n)\left(2^{2 n}-1\right)\left|B_{2 n}\right|-4\left(2^{2 n+2}-1\right)\left|B_{2 n+2}\right|}{(n-1)\left[\left(2^{2 n+2}-1\right)\left|B_{2 n+2}\right|-(n+1)\left(2^{2 n}-1\right)\left|B_{2 n}\right|\right]} \\
& >\frac{(n+2)(2 n+2)\left(2^{2 n+2}-1\right)\left|B_{2 n+2}\right|-4\left(2^{2 n+4}-1\right)\left|B_{2 n+4}\right|}{n\left[\left(2^{2 n+4}-1\right)\left|B_{2 n+4}\right|-(n+2)\left(2^{2 n+2}-1\right)\left|B_{2 n+2}\right|\right]} \\
& :=\frac{X}{Y} .
\end{aligned}
$$

Since

$$
\begin{aligned}
\frac{L Y-}{2\left|B_{2 n+2}\right|^{2}} \\
=-(n+2)\left(n^{2}-2 n-1\right)\left(2^{2 n+2}-1\right)^{2} \\
\quad-\left(2^{2 n+2}-1\right)\left(2^{2 n}-1\right)(n+2)(n+1) \frac{\left|B_{2 n}\right|}{\left|B_{2 n+2}\right|} \\
\quad+(n+1)\left(n^{2}-2 n+2\right)\left(2^{2 n+4}-1\right)\left(2^{2 n}-1\right) \frac{\left|B_{2 n}\right|}{\left|B_{2 n+2}\right|} \frac{\left|B_{2 n+4}\right|}{\left|B_{2 n+2}\right|} \\
\quad-2\left(2^{2 n+4}-1\right)\left(2^{2 n+2}-1\right) \frac{\left|B_{2 n+4}\right|}{\left|B_{2 n+2}\right|},
\end{aligned}
$$

by Lemma 5 we have

$$
\begin{aligned}
\frac{L Y-}{2\left|B_{2 n+2}\right|^{2}} & \\
> & -(n+2)\left(n^{2}-2 n-1\right)\left(2^{2 n+2}-1\right)^{2} \\
& -\frac{\left(2^{2 n+2}-1\right)\left(2^{2 n}-1\right)(n+2)(n+1) \pi^{2}\left(2^{2 n+1}-1\right)}{(2 n+2)(2 n+1)\left(2^{2 n-1}-1\right)} \\
& +(n+1)\left(n^{2}-2 n+2\right)\left(2^{2 n+4}-1\right)\left(2^{2 n}-1\right) \\
& \times \frac{\pi^{2}\left(2^{2 n+2}-1\right)}{(2 n+2)(2 n+1)\left(2^{2 n}-1\right)} \frac{(2 n+4)(2 n+3)\left(2^{2 n+1}-1\right)}{\pi^{2}\left(2^{2 n+3}-1\right)}
\end{aligned}
$$




$$
\begin{aligned}
& -2\left(2^{2 n+4}-1\right)\left(2^{2 n+2}-1\right) \frac{(2 n+4)(2 n+3)\left(2^{2 n+2}-1\right)}{\pi^{2}\left(2^{2 n+4}-1\right)} \\
= & \frac{\left(2^{2 n+2}-1\right)(n+2)}{\pi^{2}\left(2^{2 n+3}-1\right)\left(2^{2 n}-2\right)(2 n+1)} r(n),
\end{aligned}
$$

where

$$
r(n)=\left[u_{1}(n) 2^{2 n}-v_{1}(n)\right] 2^{4 n}+u_{2}(n) 2^{2 n}-v_{2}(n)
$$

with

$$
\begin{aligned}
u_{1}(n)= & \left(64 \pi^{2}-512\right) n^{2}-\left(1024-64 \pi^{2}\right) n+224 \pi^{2}-16 \pi^{4}-384, \\
v_{1}(n)= & 12 \pi^{2} n^{3}+\left(146 \pi^{2}-1216\right) n^{2}-\left(2432-140 \pi^{2}\right) n \\
& +\left(568 \pi^{2}-26 \pi^{4}-912\right), \\
u_{2}(n)= & 24 \pi^{2} n^{3}-\left(400-38 \pi^{2}\right) n^{2}-\left(800-26 \pi^{2}\right) n \\
& +247 \pi^{2}-11 \pi^{4}-300, \\
v_{2}(n)= & \left(4 \pi^{2}-32\right) n^{2}+\left(64-4 \pi^{2}\right) n-\left(14 \pi^{2}-\pi^{4}-24\right) .
\end{aligned}
$$

Then we have $c_{n} / d_{n}>c_{n+1} / d_{n+1}$ for $n \geq 3$ when proving

$$
\begin{aligned}
& u_{1}(n) 2^{2 n}-v_{1}(n)>0 \quad \Longleftrightarrow \quad 2^{2 n}>\frac{v_{1}(n)}{u_{1}(n)}, \\
& u_{2}(n) 2^{2 n}-v_{2}(n)>0 \quad \Longleftrightarrow \quad 2^{2 n}>\frac{v_{2}(n)}{u_{2}(n)} .
\end{aligned}
$$

Now we use mathematical induction to prove (2.7). When $n=3$, (2.7) clearly holds. Assuming that (2.7) holds for $n=m$, that is,

$$
2^{2 m}>\frac{v_{1}(m)}{u_{1}(m)}
$$

Next, we prove that (2.7) is valid for $n=m+1$. By (2.9) we have

$$
2^{2(m+1)}=4 \cdot 2^{2 m}>4 \frac{v_{1}(m)}{u_{1}(m)},
$$

in order to complete the proof of (2.7) it suffices to show that

$$
4 \frac{v_{1}(m)}{u_{1}(m)}>\frac{v_{1}(m+1)}{u_{1}(m+1)} \Longleftrightarrow 4 v_{1}(m) u_{1}(m+1)-v_{1}(m+1) u_{1}(m)>0 .
$$

In fact,

$$
4 v_{1}(m) u_{1}(m+1)-v_{1}(m+1) u_{1}(m)=k(m),
$$

where

$$
\begin{aligned}
k(m)= & \left(13,452,288 \pi^{4}-184,343,040 \pi^{2}-230,976 \pi^{6}+1248 \pi^{8}+728,082,432\right) \\
& +\left(11,200,896 \pi^{4}-166,279,680 \pi^{2}-103,872 \pi^{6}+663,994,368\right)(m-3)
\end{aligned}
$$




$$
\begin{aligned}
& +\left(3,983,424 \pi^{4}-59,525,376 \pi^{2}-16,608 \pi^{6}+225,067,008\right)(m-3)^{2} \\
& +\left(738,048 \pi^{4}-10,274,304 \pi^{2}-576 \pi^{6}+33,619,968\right)(m-3)^{3} \\
& +\left(68,736 \pi^{4}-801,792 \pi^{2}+1,867,776\right)(m-3)^{4} \\
& +\left(2304 \pi^{4}-18,432 \pi^{2}\right)(m-3)^{5} \\
& >0
\end{aligned}
$$

for $m \geq 3$.

Similarly, we can prove (2.8). By (2.7) and (2.8) we find that $\left\{c_{n} / d_{n}\right\}_{n \geq 3}$ is a monotonic decreasing sequence. Then we arrive at the conclusion that $p^{\prime}(x) / q^{\prime}(x)=C(x) / D(x)$ is decreasing on $(0, \pi / 2)$ by Lemma 2 . By Lemma 1 we see that $z(x)$ is decreasing on $(0, \pi / 2)$.

Since

$$
z\left(0^{+}\right)=\frac{1}{5}, \quad z\left(\left(\frac{\pi}{2}\right)^{-}\right)=0,
$$

this completes the proof of Lemma 6 .

\section{The proofs of main results}

\subsection{The proof of Theorem 4}

Proof Let

$$
G(x)=\frac{2 \cosh ^{p} x+1}{3 e^{p(x \operatorname{coth} x-1)}}, \quad x>0 .
$$

Then

$$
G(+\infty)= \begin{cases}\frac{2}{3}\left(\frac{e}{2}\right)^{p}, & p>0 \\ +\infty, & p<0\end{cases}
$$

and

$$
G^{\prime}(x)=\frac{p}{3} \frac{Q(x)}{e^{p(x \operatorname{coth} x-1)}},
$$

where

$$
\begin{aligned}
Q(x)= & \frac{2(x \cosh x-\sinh x)}{\sinh ^{2} x} \cosh ^{p-1} x-\frac{\cosh x \sinh x-x}{\sinh ^{2} x} \\
= & \frac{2(x \cosh x-\sinh x)}{\sinh ^{2} x}\left[\cosh ^{p-1} x-\frac{\cosh x \sinh x-x}{2(x \cosh x-\sinh x)}\right] \\
= & \frac{2(x \cosh x-\sinh x)(\ln \cosh x)}{\sinh ^{2} x} \frac{\cosh ^{p-1} x-\frac{\cosh x \sinh x-x}{2(x \cosh x-\sinh x)}}{\ln \left(\cosh ^{p-1} x\right)-\ln \frac{\cosh x \sinh x-x}{2(x \cosh x-\sinh x)}} \\
& \times\left(p-1-\frac{\ln \frac{\sinh 2 x-2 x}{4(x \cosh x-\sinh x)}}{\ln \cosh x}\right)
\end{aligned}
$$




$$
\begin{aligned}
=: & 2 \frac{(x \cosh x-\sinh x)(\ln \cosh x)}{\sinh ^{2} x} \frac{\cosh ^{p-1} x-\frac{\cosh x \sinh x-x}{2(x \cosh x-\sinh x)}}{\ln \left(\cosh ^{p-1} x\right)-\ln \frac{\cosh x \sinh x-x}{2(x \cosh x-\sinh x)}} \\
& \times(p-1-l(x))
\end{aligned}
$$

with

$$
l(x)=\frac{\ln \frac{\sinh 2 x-2 x}{4(x \cosh x-\sinh x)}}{\ln \cosh x}
$$

We consider the following three cases.

Case 1: $p \geq 2$.

From Lemma 3 , we get $\max _{x \in(0,+\infty)} l(x)=1$. So $p-1-l(x)>0$. This leads to $Q(x)>0$ by (3.2), and $G^{\prime}(x)>0$ by (3.1). Then

$$
G\left(0^{+}\right)<G(x)<G(+\infty)
$$

this is the double inequality (1.5).

Case 2: $p \leq 6 / 5$.

From Lemma 3, we get $\min _{x \in(0,+\infty)} l(x)=1 / 5$. So $p-1-l(x)<0$. This leads to $Q(x)<0$.

Subcase 2.1: $0<p \leq 6 / 5$. In this case, $G^{\prime}(x)<0$ by (3.1). Then

$$
G(+\infty)<G(x)<G\left(0^{+}\right)
$$

this is the double inequality (1.6).

Subcase 2.2: $p<0$. We have $G^{\prime}(x)>0$ by (3.1). In view of $G(+\infty)=+\infty$, the left-hand side of inequality (1.5) holds too.

Case 3: $6 / 5<p<2$.

Let $r(x):=l(x)+1-p$. Then

$$
\begin{aligned}
& r\left(0^{+}\right)=l\left(0^{+}\right)+1-p=\frac{6}{5}-p<0, \\
& r(+\infty)=l(+\infty)+1-p=2-p>0,
\end{aligned}
$$

and there is the unique point $\xi \in(0,+\infty)$ such that $r(x)<0$ holds for all $x \in(0, \xi)$ and $r(x)>0$ holds for all for $x \in(\xi,+\infty)$. That is, $p-1-l(x)>0$ holds for all $x \in(0, \xi)$ and $p-1-l(x)>0$ holds for all $x \in(\xi,+\infty)$. By (3.2) and (3.1), we have $G^{\prime}(x)>0$ for all $x \in(0, \xi)$ and $G^{\prime}(x)<0$ holds for all $x \in(\xi,+\infty)$. Then

$$
\min \left(G\left(0^{+}\right), G(+\infty)<G(x)<G(\xi) .\right.
$$

Subcase 3.1: $p_{1}=(\ln (3 / 2)) /(\ln (e / 2))<p<2$. In this case, $1<(2 / 3)(e / 2)^{p}$, that is, $G\left(0^{+}\right)<$ $G(+\infty)$ holds, so $\min \left(G\left(0^{+}\right), G(+\infty)\right)=G\left(0^{+}\right)$. This leads to the left-hand side of inequality (1.5).

Subcase 3.2: $6 / 5<p<p_{1}=(\ln (3 / 2)) /(\ln (e / 2))$. In this case, $1>(2 / 3)(e / 2)^{p}$, that is, $G\left(0^{+}\right)>$ $G(+\infty)$ holds, so $\min \left(G\left(0^{+}\right), G(+\infty)\right)=G(+\infty)$. This leads to the left-hand side of inequality (1.6).

The proof of Theorem 4 is complete. 


\subsection{The proof of Theorem 6}

Proof Let

$$
F(x)=\frac{2 \cos ^{p} x+1}{3 e^{p(x \cot x-1)}}, \quad 0<x<\frac{\pi}{2} .
$$

Then

$$
F\left(\left(\frac{\pi}{2}\right)^{-}\right)=\frac{e^{p}}{3}
$$

and

$$
\begin{aligned}
F^{\prime}(x)= & \frac{p}{3} \frac{2(\sin x-x \cos x)[-\ln (\cos x)]}{\left(\sin ^{2} x\right) \exp (p(x \cot x-1))} \frac{\cos ^{p-1} x-\frac{x-\cos x \sin x}{2(\sin x-x \cos x)}}{(p-1) \ln \cos x-\ln \frac{(x-\cos x \sin x)}{2(\sin x-x \cos x)}} \\
& \times\left(p-1-\frac{\ln \frac{2 x-\sin 2 x}{4(\sin x-x \cos x)}}{\ln \cos x}\right) \\
= & \frac{p}{3} \frac{2(\sin x-x \cos x)[-\ln (\cos x)]}{\left(\sin ^{2} x\right) \exp (p(x \cot x-1))} \frac{\cos ^{p-1} x-\frac{x-\cos x \sin x}{2(\sin x-x \cos x)}}{(p-1) \ln \cos x-\ln \frac{(x-\cos x \sin x)}{2(\sin x-x \cos x)}} \\
& \times[p-1-z(x)],
\end{aligned}
$$

where

$$
z(x)=\frac{\ln \frac{2 x-\sin 2 x}{4(\sin x-x \cos x)}}{\ln \cos x} .
$$

We consider the following three cases.

Case $1: p \geq 6 / 5$.

From Lemma 6, we get $\max _{x \in(0,+\infty)} z(x)=1 / 5$. So $p-1-z(x)>0$ holds. This leads to $F^{\prime}(x)<0$ by (3.3). Then

$$
F\left(0^{+}\right)<F(x)<F\left(\left(\frac{\pi}{2}\right)^{-}\right),
$$

which is the double inequality (1.8).

Case 2: $p \leq 1$.

From Lemma 6, we get $\min _{x \in(0,+\infty)} z(x)=0$. So $p-1-z(x)<0$ holds.

Subcase 2.1: $0<p \leq 1$. In this case, $F^{\prime}(x)<0$ by (3.3). Then

$$
F\left(\left(\frac{\pi}{2}\right)^{-}\right)<F(x)<F\left(0^{+}\right)
$$

which is the double inequality (1.9).

Subcase 2.2: $p<0$. We have $F^{\prime}(x)>0$ by (3.3), the double inequality (1.8) holds too.

Case 3: $1<p<6 / 5$.

Let $q(x):=z(x)-p+1$. Then $q\left(0^{+}\right)=z\left(0^{+}\right)-p+1=6 / 5-p>0, q\left((\pi / 2)^{-}\right)=z\left((\pi / 2)^{-}\right)-p+$ $1=1-p<0$. There is a unique point $\eta \in(0, \pi / 2)$ such that $q(x)>0$ holds for all $x \in(0, \eta)$ 
and $q(x)<0$ holds for all for $x \in(\eta, \pi / 2)$. That is, $p-1-z(x)<0$ holds for all $x \in(0, \eta)$ and $p-1-z(x)>0$ holds for all $x \in(\eta, \pi / 2)$. By (3.3), we have $F^{\prime}(x)<0$ for all $x \in(0, \eta)$ and $F^{\prime}(x)>0$ for all $x \in(\eta, \pi / 2)$. Then

$$
F(\eta)<F(x)<\max \left(F\left(0^{+}\right), F\left((\pi / 2)^{-}\right)\right)
$$

Subcase 3.1: $p_{2}=\ln 3=1.0986<p<6 / 5$. In this case, $1<e^{p} / 3$, that is, $F\left(0^{+}\right)<F\left((\pi / 2)^{-}\right)$, so $\max \left(F\left(0^{+}\right), F\left((\pi / 2)^{-}\right)\right)=F\left((\pi / 2)^{-}\right)$. This leads to the right-hand side of inequality (1.8).

Subcase 3.2: $1<p \leq p_{2}=\ln 3=1.0986$. In this case, $1 \geq e^{p} / 3$, that is, $F\left(0^{+}\right) \geq F\left((\pi / 2)^{-}\right)$, so $\max \left(F\left(0^{+}\right), F\left((\pi / 2)^{-}\right)\right)=F\left(0^{+}\right)$. This leads to the right-hand side of inequality (1.9).

The proof of Theorem 6 is complete.

\section{Acknowledgements}

The author is thankful to reviewers for their careful corrections to and valuable comments on the original version of this paper.

\section{Funding}

The author was supported by the National Natural Science Foundation of China (no. 61772025).

\section{Competing interests}

The author declares that he has no competing interests.

\section{Authors' contributions}

The author provided the questions and gave the proof for all results. He read and approved this manuscript.

\section{Publisher's Note}

Springer Nature remains neutral with regard to jurisdictional claims in published maps and institutional affiliations.

Received: 6 March 2019 Accepted: 7 August 2019 Published online: 20 August 2019

\section{References}

1. Stolarsky, K.B.: Generalizations of the logarithmic mean. Math. Mag. 48(2), 87-92 (1975). https://doi.org/10.1080/0025570x.1975.11976447

2. Pittenger, A.O.: Inequalities between arithmetic and logarithmic means. Publ. Elektroteh. Fak. Univ. Beogr., Ser. Mat. Fiz. 678(715), 15-18 (1980)

3. Stolarsky, K.B.: The power and generalized logarithmic means. Am. Math. Mon. 87(7), 545-548 (1980). https://doi.org/10.1080/00029890.1980.11995086

4. Zhu, L.: Some new inequalities for means in two variables. Math. Inequal. Appl. 11(3), 443-448 (2008) https://doi.org/10.7153/mia-11-33

5. Kouba, O.: New bounds for the identric mean of two variables. J. Inequal. Pure Appl. Math. 9(3), 71 (2008)

6. Zhu, L.: Sharp inequalities for hyperbolic functions. Appl. Math. Comput. 219(1), 416-420 (2012). https://doi.org/10.1016/j.amc.2012.06.035

7. Yang, Z.-H., Jiang, Y.L., Song, Y.Q., Chu, Y.M.: Sharp inequalities for trigonometric functions. Abstr. Appl. Anal. 2014, Article ID 601839 (2014) https://doi.org/10.1155/2014/601839

8. Anderson, G.D., Vamanamurthy, M.K., Vuorinen, M.: Conformal Invariants, Inequalities, and Quasiconformal Maps, Wiley, New York (1997)

9. Biernacki, M., Krzyz, J.: On the monotonicity of certain functionals in the theory of analytic functions. Ann. Univ. Mariae Curie-Skłodowska 9, 135-147 (1955)

10. Ireland, K., Rosen, M.: A Classical Introduction to Modern Number Theory, 2nd edn. Springer, New York (1990)

11. Jeffrey, A.: Handbook of Mathematical Formulas and Integrals, 3rd edn. Elsevier, Amsterdam (2004)

12. Qi, F:: A double inequality for the ratio of two non-zero neighbouring Bernoulli numbers. J. Comput. Appl. Math. 351, 1-5 (2019). https://doi.org/10.1016/j.cam.2018.10.049

13. Qi, F.: Notes on a double inequality for ratios of any two neighbouring non-zero Bernoulli numbers. Turk. J. Anal. Number Theory 6(5), 129-131 (2018). https://doi.org/10.12691/tjant-6-5-1 